\title{
An endogenous peptide marker differentiates SOD1 stability and facilitates pharmacodynamic monitoring in SOD1 amyotrophic lateral sclerosis
}

\author{
Ilya Gertsman, ${ }^{1,2}$ Joanne Wuu, ${ }^{3}$ Melissa McAlonis-Downes, ${ }^{4}$ Majid Chassemian, ${ }^{5}$ Karen Ling, ${ }^{6}$ \\ Frank Rigo, ${ }^{6}$ Frank Bennett, ${ }^{6}$ Michael Benatar, ${ }^{3}$ Timothy M. Miller, ${ }^{7}$ and Sandrine Da Cruz ${ }^{4}$ \\ 'Biochemical Genetics and Metabolomics Laboratory, Department of Pediatrics, UCSD, La Jolla, California, USA. '2Clarus \\ Analytical, LLC, San Diego, California, USA. ${ }^{3}$ Department of Neurology, University of Miami, Miami, Florida, USA. ${ }^{4}$ Ludwig \\ Institute for Cancer Research and ${ }^{5}$ Biomolecular/Proteomics Mass Spectrometry Facility, Department of Chemistry and \\ Biochemistry, UCSD, La Jolla, California, USA. ' Ionis Pharmaceuticals, Carlsbad, California, USA. 'Department of Neurology, \\ Washington University in St. Louis, St. Louis, Missouri, USA.
}

The discovery of novel biomarkers has emerged as a critical need for therapeutic development in amyotrophic lateral sclerosis (ALS). For some subsets of ALS, such as the genetic superoxide dismutase 1 (SOD1) form, exciting new treatment strategies, such as antisense oligonucleotidemediated (ASO-mediated) SOD1 silencing, are being tested in clinical trials, so the identification of pharmacodynamic biomarkers for therapeutic monitoring is essential. We identify increased levels of a 7-amino acid endogenous peptide of SOD1 in cerebrospinal fluid (CSF) of human SOD1 mutation carriers but not in other neurological cases or nondiseased controls. Levels of peptide elevation vary based on the specific SOD1 mutation (ranging from 1.1-fold greater than control in D90A to nearly 30 -fold greater in V148C) and correlate with previously published measurements of SOD1 stability. Using a mass spectrometry-based method (liquid chromatography-mass spectrometry), we quantified peptides in both extracellular samples (CSF) and intracellular samples (spinal cord from rat) to demonstrate that the peptide distinguishes mutation-specific differences in intracellular SOD1 degradation. Furthermore, $80 \%$ and $63 \%$ reductions of the peptide were measured in SOD1 ${ }^{193 A}$ and SOD1 ${ }^{\text {H46R }}$ rat CSF samples, respectively, following treatment with ASO, with an improved correlation to mRNA levels in spinal cords compared with the ELISA measuring intact SOD1 protein. These data demonstrate the potential of this peptide as a pharmacodynamic biomarker.

Conflict of interest: IC and SDC are listed as coinventors on patent application 62/822,692 related to the biomarker described in this manuscript. TMM is a member of the medical advisory board of Biogen and receives support for clinical studies from Biogen; has a licensing agreement and receives material support from lonis Pharmaceuticals; has a licensing agreement with $\mathrm{C} 2 \mathrm{~N}$; and is a consultant for Cytokinetics.

Copyright: (c) 2019 American Society for Clinical Investigation

Submitted: August 6, 2018

Accepted: April 4, 2019

Published: May 16, 2019.

Reference information: JCI Insight. 2019;4(10):e122768. https://doi. org/10.1172/jci.insight.122768

\section{Introduction}

Amyotrophic lateral sclerosis (ALS) is the most common adult-onset motor neuron disease, causing rapid degeneration in motor function. About $10 \%$ of the cases are familial (1), including genetic causes such as hexanucleotide expansion repeats in c9orf72 $(2,3)$, mutations in DNA- and RNA-binding proteins like TDP-43 $(4,5)$ and FUS $(6,7)$, as well as the most well-studied inherited cause, that of superoxide dismutase 1 (SOD1), which represents $20 \%$ of all familial cases in some populations (SOD1 ALS cases) $(8,9)$. A common feature of ALS is misfolding or aggregation of polypeptides or proteins that deposit in motor neurons and may indeed be the root cause of cell toxicity. There have been approximately 150 genetic mutations identified in SOD1 that cause ALS, varying in their rates of disease progression and severity and partially correlating with the degree of misfolding or aggregation (10).

Unlike the majority of ALS cases in which the cause of disease is unknown, genetic forms, such as those associated with SOD1, provide a clearer target for therapeutic intervention. Recently targeted strategies for diseased protein in neurodegenerative disease have led to several drug technologies that are currently being tested in ALS (11). These include antisense oligonucleotide (ASO) therapy and viral-delivered siRNA strategies, shown to be successful in reducing SOD1 and delaying disease progression in animal models and currently being evaluated in clinical trials (12-15). Another therapeutic approach is the use of the drug Arimoclomol, an 
activator of heat shock proteins (HSPs) that facilitate increased protein folding and stabilization in response to cell damage and stress, previously shown to be diminished in mouse models of ALS $(16,17)$. Arimoclomol has shown promising neuroprotective properties in experiments with mice and is currently being tested in clinical trials (17-20). In addition to establishing improved treatments for SOD1 ALS, it will be valuable to find pharmacodynamic markers that are useful for evaluating the various treatment strategies under investigation. One such validated assay involves measuring SOD1 protein in CSF, shown to decrease approximately $40 \%$ upon treatment with ASOs in SOD1 ${ }^{\mathrm{G} 93 \mathrm{~A}}$ rats (21). There is no current assay, though, that can distinguish differences in stability of SOD1, which can lead to protein misfolding, a critical component of the disease and potentially an important marker for current therapeutic strategies.

We investigated biomarkers in ALS using untargeted metabolomic analysis of cerebrospinal fluid (CSF), initially in established ALS rat models expressing mutant SOD1 proteins with markedly different biochemical characteristics, including dismutase-active SOD $1^{\mathrm{G} 93 \mathrm{~A}}$ and -inactive SOD $1^{\mathrm{H} 46 \mathrm{R}}(22)$. Increased levels ( 10-fold) of an endogenous peptide of SOD1 were discovered and further tested in human CSF of SOD1 mutation carriers and controls using a quantitative assay developed for this peptide. We observed varying degrees of peptide elevation that reflect mutation-specific differences in SOD1 stability and breakdown. We also demonstrate using ASO-treated rats the peptide's potential utility as a pharmacodynamic biomarker for SOD1-targeted drugs.

Peptides have previously been shown to be elevated in other neurological disorders, such as Alzheimer's disease, specifically tau and a fragment of amyloid- $\beta$ protein (23), but no studies to our knowledge have investigated small endogenous peptide products from intracellular protein turnover mechanisms (e.g., proteasome or autophagy) as a monitor for aberrant protein misfolding and processing in disease states. We believe the assay described here can assess these properties for SOD1 mutation carriers, relevant for both improved understanding of the role of SOD1 in disease, as well as for SOD1-targeted therapies currently under investigation.

\section{Results}

Biomarker discovery in SOD1 rats using liquid chromatography-mass spectrometry. Our initial search for biomarkers in ALS was performed with CSF samples from the mutant SOD1 ${ }^{\mathrm{G} 93 \mathrm{~A}}$ rat model $(n=9)$ and age-matched animals expressing human SOD $1^{\mathrm{WT}}$ using a previously validated metabolomics platform (24). Although there were small changes in a handful of metabolites, a nearly 10 -fold change in 2 analytes $(\mathrm{m} / z$ of 351.195 and 307.681) that eluted approximately 0.2 minutes from each other was detected (Figure 1A). We measured a similar elevation in a second mutant SOD1 rat model (SOD1 ${ }^{\mathrm{H} 46 \mathrm{R}}, n=4$ rats), whose expression of dismutase-inactive, SOD1-mutant protein causes age-dependent paralytic disease (Figure 1B). Analysis of the charge state, chromatographic elution time, and tandem mass spectrometry (MS/MS) patterns suggested that these compounds were peptides. The former peptide $351 \mathrm{~m} / z$ had a greater, more reliable overall signal, and so we proceeded with absolute identification of this peptide, whose sequence was found to be SDGPVKV by matching both the fragmentation pattern (MS/MS) and chromatographic elution profile with that of a custom-synthesized peptide (Supplemental Figure 1; supplemental material available online with this article; https://doi.org/10.1172/jci. insight.122768DS1). From these data, we also identified the sequence of the $308 \mathrm{~m} / z$ ion to be DGPVKV, which is the sequence of peptide $351 \mathrm{~m} / z$ missing the N-terminal serine (Supplemental Figure 2).

The matched sequences were searched against both rat and human genomic databases using the protein BLAST search tool (National Center for Biotechnology Information). The only feasible match for the larger peptide was the sequence SNGPVKV from human SOD1, with a single D $>\mathrm{N}$ substitution, a common occurrence in proteins because of $\mathrm{N}$ deamidation. The $\mathrm{D}$ form carries a mass difference of $1.007 \mathrm{amu}$, so it could not be misconstrued with the $\mathrm{N}$ form using our high-resolution mass spectrometry. We used a synthesized $\mathrm{N}$ version of the peptide to ensure there was no preparative- or instrument-induced deamidation (Supplemental Figure 1D).

In addition to elevations in CSF, we observed increased intracellular accumulation of the endogenous peptide in total spinal cord homogenates from SOD $1^{\mathrm{G} 93 \mathrm{~A}}$ compared with SOD $1^{\mathrm{WT}}$ rats (Figure 1C). It is also important to note that this increase was not caused by elevation of intact SOD1 protein in spinal cords, which we show was similar in SOD $1^{\mathrm{G} 93 \mathrm{~A}}$ and even diminished in SOD $1^{\mathrm{H} 46 \mathrm{R}}$ (Figure 1D), thus suggesting a unique or increased intracellular SOD1 turnover process occurring in the SOD1 mutants.

Translation of biomarker measurement to human CSF samples. We measured the same $351 \mathrm{~m} / z$ peptide in human CSF, but the $308 \mathrm{~m} / z$ peptide was not detected. We therefore focused on the $351 \mathrm{~m} / z$ peptide in further studies (termed "SOD1 peptide" in this manuscript) and measured its concentration in 27 
A

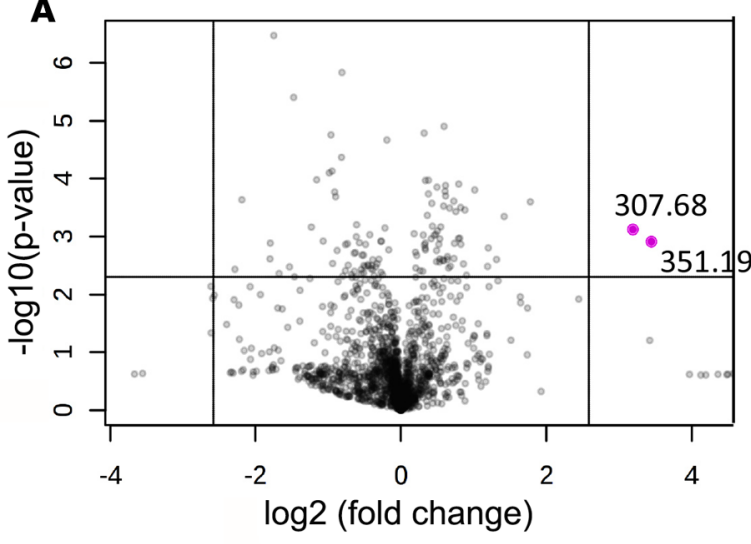

C

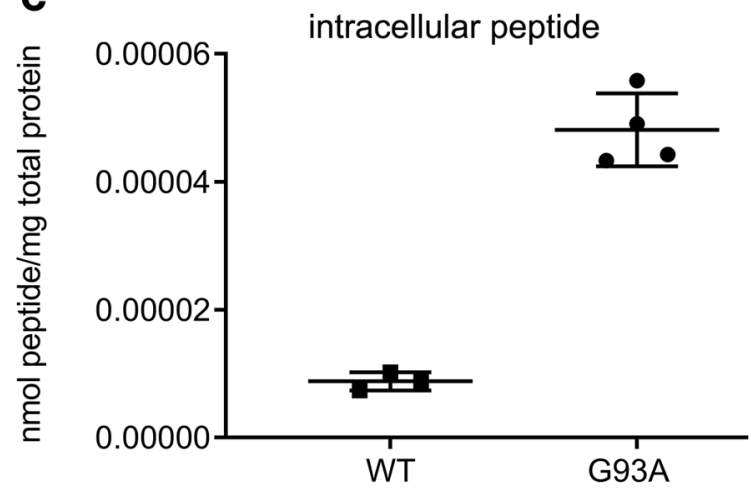

B

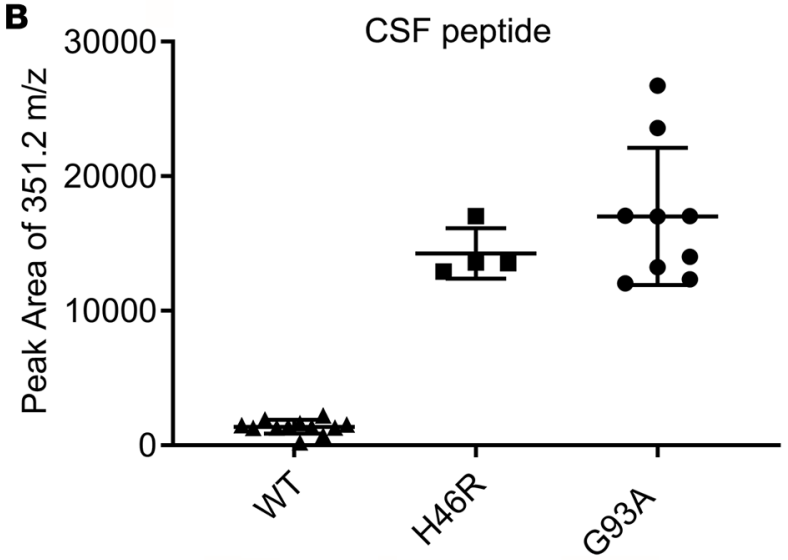

D

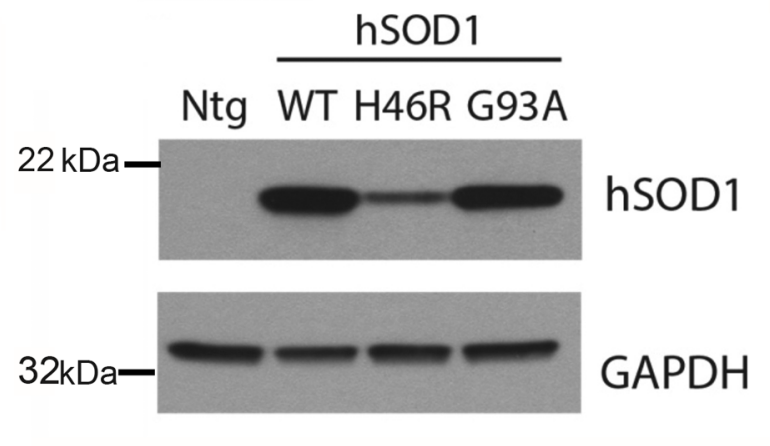

Figure 1. Discovery of elevated peptides in CSF of SOD1 rats. (A) Volcano plot from untargeted metabolomics analysis comparing SOD1 ${ }^{\mathrm{C93A}}$ rats with SOD1WT rats. Two analytes (shown in magenta) were significantly elevated, approximately 12 -fold $(P<0.0001)$, and later identified as peptides of SOD1. (B) Dot plots

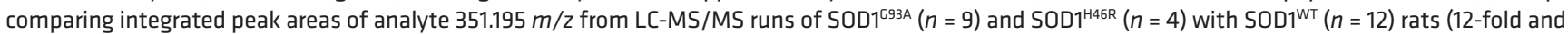
10-fold change compared with WT respectively; $P<0.0001$ from Mann-Whitney $U$ test). (C) SOD1 peptide ( $m / z$ 351.195) concentration determined in spinal cord homogenates (WT: $n=3$; G93A: $n=4$; fold change: 5.5; $P<0.05$ ). (D) Immunoblot analysis, showing intact SOD1 levels in spinal cords of symptomatic

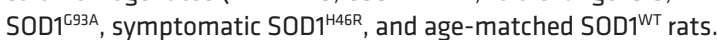

human CSF samples from SOD1 mutation carriers ( $n=14$ ALS patients, and $n=13$ presymptomatic gene mutation carriers), 29 controls (with no known neurological disease), and 30 neurological disease controls (Alzheimer's disease and multiple sclerosis) using a liquid chromatography-mass spectrometry (LC-MS/ MS) method developed for absolute quantification (i.e., standard curve generated for each batch and stable isotope dilution for internal standard use). The mass spectrometry analysis of all mutation carriers and control samples was executed by an observer who was blinded to the sample identity. All SOD1 variants were elevated compared with both control and disease controls, ranging from only 1.1-fold in D90A to as much as 30-fold for V148G (Figure 2 and Table 1). A4V, the most common SOD1 variant in North America, had 5 unique patient samples measured, with a 9-fold elevation from controls, with only a $16 \%$ $\mathrm{SD}$ within the cohort $(224.6 \pm 43.0$ picomolars $[\mathrm{pM}])$. The lone A4T sample had nearly identical peptide levels as the $\mathrm{A} 4 \mathrm{~V}$ samples. It is interesting to note that the only recessive form, D90A, exhibited the least amount of peptide, and the A89V mutation, which exhibits incomplete penetrance, was also on the lower end of the fold elevation among the variants measured. The $2 \mathrm{~A} 89 \mathrm{~V}$ samples tested were acquired from the same patient but 4.1 years apart. V148G, an aggressive variant (2.1 years average disease duration; ref. $10)$, showed the highest levels $(755.0 \pm 137.2 \mathrm{pM})$ of peptide, which were measured in presymptomatic mutation carriers. Notably, a third V148G sample was analyzed from an ALS patient treated with Arimoclomol as part of a clinical trial, and the result was a considerably lower peptide concentration (250.0 pM). Overall, although the range of the SOD1 peptide concentrations varied extensively among the different SOD1 variants, the increased concentration was mostly specific to the SOD1 ALS mutation, exhibiting a low intracohort deviation $(<20 \% \mathrm{SD})$ for the 5 out of 7 mutations for which multiple patient samples could 
A

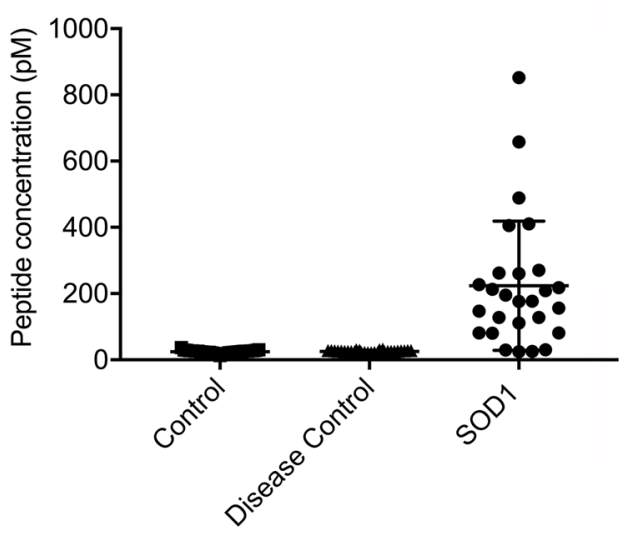

B

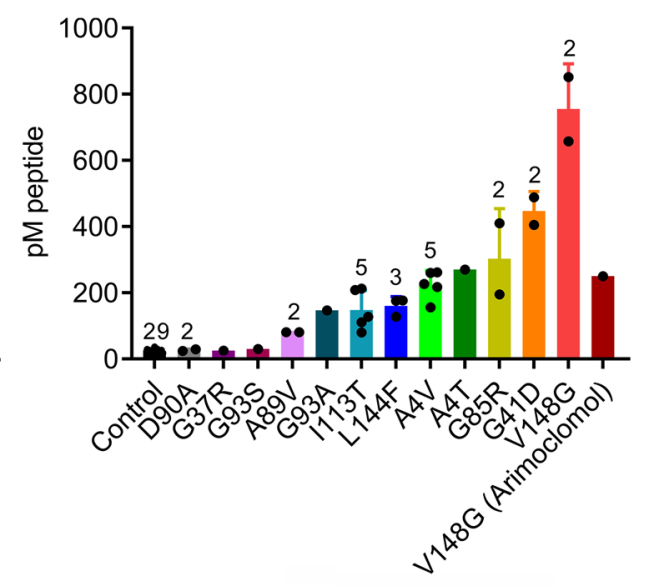

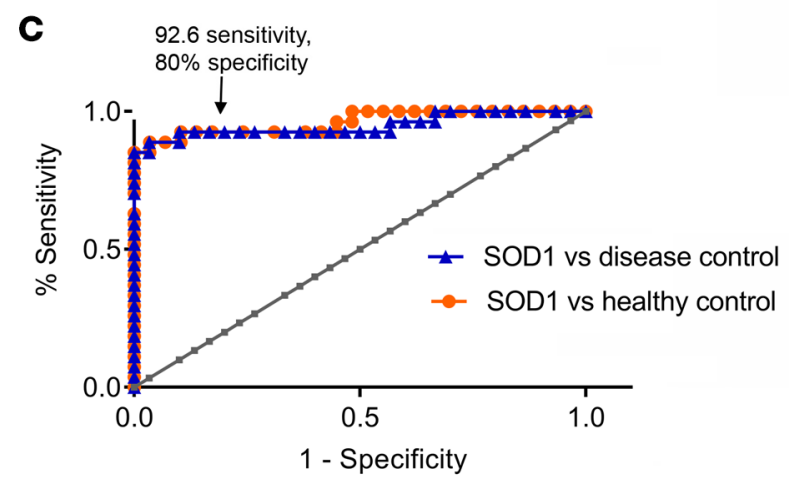

Figure 2. SOD1 peptide in human CSF samples. (A) Comparison between 29 control samples, 30 disease control samples, and 27 SOD1 samples. There was a 9 -fold difference (Mann-Whitney $U$ test, $P<0.0001$ ) between the SOD1 group and both control groups (scatter plot showing bars for mean and SD). (B) Bar graphs of peptide concentrations for the different genetic variants of SOD1 (individual measurements represented as dots). The number of unique samples of a given variant is noted above the bar when 2 or greater, and error bars denote standard deviation. (C) Receiver operator characteristic (ROC) curve comparing SOD1 with control and disease control groups (area under the curve of 0.95 and 0.96 , respectively).

be tested. The accuracy of this marker in distinguishing carriers of SOD1-linked mutations to both control and disease control cohorts was assessed using a ROC curve (Figure 2C). The assay has high overall specificity and sensitivity for ALS-associated SOD1 mutations (sensitivity of $92.6 \%$ at $80 \%$ specificity in both comparisons, area under the curve of 0.96 for SOD1 analyzed against control and 0.95 against diseased control, 95\% confidence interval of $0.89-1.01, P<0.0001$ ).

Tracing the origin of the endogenous peptide. An important aspect to assess was whether deamidation was occurring in the native protein state and whether it was greater in mutant SOD1 than WT. Deamidation of native protein often occurs in aged protein but can also be more abundant in regions of proteins that are more dynamic (lower order of structure) or misfolded (25). We investigated native SOD1 protein deamidation at the same amino acid position (ASN 26) from spinal cords of SOD $1^{\mathrm{G} 93 \mathrm{~A}}$ rats and compared these to control human SOD $1^{\mathrm{WT}}$ rats. No difference in percentage of deamidation of native SOD1 was observed between SOD ${ }^{\mathrm{WT}}$ and SOD $1^{\mathrm{G} 93 \mathrm{~A}}$ homogenates, observed at levels of less than $10 \%$ for both groups (Supplemental Figure 3 ). This result demonstrated that the cleaved peptide product is therefore deamidated primarily after proteolysis, rather than in the intact protein state. Our findings of postproteolytic deamidation are in agreement with research highlighting that asparagines in unfolded proteins or peptides undergo significant deamidation endogenously (26), especially those that neighbor a glycine residue as is the case in the SOD1 peptide we identified.

We used an in silico prediction tool (NetChop20S-3.0; ref. 27) to determine the likelihood of this peptide resulting from ubiquitin-based proteasomal cleavage. The $20 \mathrm{~S}$ proteasome has previously been linked to mutant SOD1 degradation in ALS (28). The analysis, using predictive 20S proteasomal degradation of the SOD1 protein 
sequence, identified that the amino acid $\mathrm{N}$-terminal to the peptide (E24), as well as the C-terminal residue of the peptide (V31), had high likelihoods of cleavage by the proteasome (scores $>0.8$ ), whereas further internal fragments of this peptide were considerably less probable (scores all < 0.5) (Supplemental Figure 4).

Because the rate of protein turnover can be influenced by protein misfolding and decreased stability, we correlated our peptide concentrations to relevant SOD1 stability data in the literature. Lindberg et al. previously characterized SOD1 stability changes $(\Delta \Delta \mathrm{G} \mathrm{kcal} / \mathrm{mol})$ in common mutants of ALS, considering both destabilization of the free monomer, as well as the dimer interface (29). For the 8 overlapping mutants monitored by both their study and ours, we observed a significant correlation between peptide concentration and SOD1 destabilization $(\Delta \Delta G)$ in relation to WT SOD1, as shown in Figure 3 (Spearman's $r .-0.75, P<0.05$ ).

SOD1 peptide as a pharmacodynamic marker. To determine whether the SOD1 peptide could represent a pharmacodynamic biomarker for SOD1-lowering therapies, presymptomatic SOD $1^{\mathrm{G} 93 \mathrm{~A}}$ and SOD1 ${ }^{\mathrm{H} 46 \mathrm{R}}$ rats were treated with a single intraventricular bolus injection of ASOs (14) targeting human SOD1 RNA or with saline. After 6 weeks of treatment, SOD1 peptide was quantified in CSF from ASO-treated mutant SOD1 rats compared with CSF from saline-treated and untreated animals carrying the mutation (referred to as "non-ASO-treated"). An 80\% decrease in the SOD1 peptide was measured in the CSF of ASO-treated SOD $1^{\mathrm{G} 93 \mathrm{~A}}$ as compared with non-ASO-treated rats $(8178 \pm 730$ pM vs. $1661 \pm 326$ pM), while a $63 \%$ decrease was observed in ASO-treated SOD $1^{\mathrm{H} 46 \mathrm{R}}$ rats $(3445 \pm 1784 \mathrm{pM}$ vs. $1279 \pm 55 \mathrm{pM})$. Per condition, $n=4$ rats were used $(P<0.05$, Mann-Whitney $U$ test for both comparisons) (Figure 4$)$. Note that the levels of SOD1 peptide measured in CSF from non-ASO-treated SOD1 ${ }^{\mathrm{G} 93 \mathrm{~A}}$ rats were 2.4-fold higher than in the mutant SOD1 ${ }^{\mathrm{H} 46 \mathrm{R}}$ animals, possibly because of the higher levels of expression of the human transgene (Figure 1D). We compared the decrease in peptide levels with mRNA levels measured in the spinal cord, as well as the standard SOD1 ELISA used as a pharmacodynamic marker in current ASO clinical trials (Figure 4 and Table 2). In SOD1 ${ }^{\mathrm{G} 93 \mathrm{~A}}$ rats, we observed a $77 \%$ reduction in mRNA levels, very similar to the $80 \%$ reduction in peptide, while SOD 1 protein as measured by ELISA was reduced by only $32 \%$. For $\mathrm{H} 46 \mathrm{R}$, there was a similar percentage reduction in mean peptide levels as there was mRNA following ASO treatment (63\% vs. $64 \%$, respectively). The SOD1 ELISA for H46R was inconclusive and is not shown. We believe the antibody for the ELISA kit may not recognize the H46R-misfolded form of SOD1 as well as it does G93A, and because the rat model expresses only the mutant form, unlike human patients, who are typically heterozygous, the assay may not be applicable for this mutation in rats.

\section{Discussion}

We believe that the peptide product of intracellular SOD1 degradation described in this manuscript provides a simple and highly sensitive assay, using CSF, for assessing SOD1 protein stability and degradation in vivo. Because greater protein misfolding can lead to greater degradation $(30,31)$, we believe increased SOD1 peptide concentrations are correlated to the decreased stability of SOD1, which varies with the severity of the mutation. We observed a significant correlation to previously collected stability measurements of SOD1 mutants but did not observe a significant correlation with survival in our limited sampling, primarily because of outliers, such as G41D and G85R, which had high peptide levels, yet are associated with higher survival times. Lindberg et al. also reported these specific genetic outliers in their correlation with survival, hypothesized as being related to changes in net charge, which may affect aggregation and disease progression differently than a neutral amino acid substitution (29).

Interestingly, the levels of the SOD1 peptide measured in CSF were stable through disease course, with the increased fold change being similar at early and late stages of disease in SOD1-mutant rats and in the genetic variant D90A, where we measured both presymptomatic and affected patient samples (from different individuals), with little difference observed (30 pM vs. $25 \mathrm{pM}$ ). In addition, the 2 measurements of A89V CSF from the same patient staggered by nearly 4 years were nearly identical ( 80.9 pM vs. 81.3 pM). Farr et al. previously reported that the turnover rate of the soluble pool of SOD1 was identical in presymptomatic and symptomatic mice (32), which if it applies similarly in humans, could explain this lack of change during disease progression.

The data show that the peptide increase is also found intracellularly in spinal cord tissue in addition to CSF, and in silico predictions implicate the $20 \mathrm{~S}$ proteasome as a possible mechanism for this peptide product, though lysosomal degradation cannot be ruled out at this stage. Recognizing that we would not be able to measure actual turnover rates of human SOD1 variants, which would require stable isotope administration into humans (33), we compared our SOD1 peptide levels from human CSF with turnover rates 


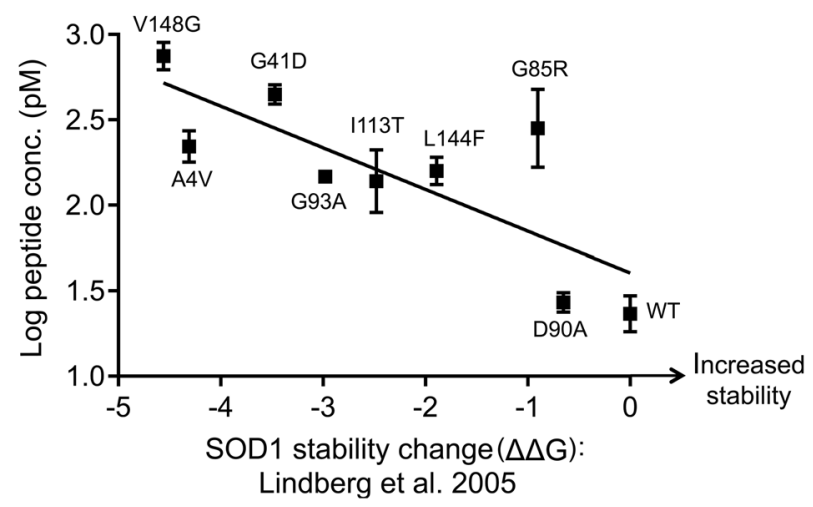

Figure 3. Correlation of endogenous peptide concentration with SOD1 stability $(\Delta \Delta \mathrm{G}$ $\mathrm{kcal} / \mathrm{mol}$ ) data from Lindberg et al. Eight mutations and WT overlapped between our own study and the Lindberg et al. study, as labeled in the graph. Correlation analysis: Spearman's $r=-0.75 ; P<0.05$.

previously described $(28,32,33)$. Di Noto et al. measured proteasomal SOD1 degradation in vitro (using purified 20S proteasome) and showed an ascending rate of turnover for 3 variants (G37R $<$ G93A $<A 4 V$ ) (28). Interestingly, we found a similar order of SOD1 peptide concentrations among these same 3 variants. To date, only 2 studies have quantified SOD1 turnover in vivo, one executed in mice and the other in rats $(32,33)$. Though strict comparisons of the previous studies cannot be done, because different types of rodents and MS-based methodologies were used, it is worth noting that differences in turnover rate (G85R was 8.5 times faster than WT as measured by Farr et al., and G93A was 1.6 times faster than WT in Crisp et al.) followed a similar order as our SOD1 peptide concentrations (G85R>G93A, Table 1) $(32,33)$. Future experiments measuring both turnover rate and SOD1 peptide levels from the same subject for a variety of mutations will be required to determine whether the peptide can be used as a surrogate for evaluating SOD1 turnover in the disease, a measurement that would be especially valuable for optimizing drug dosing and administration frequency for therapies such as ASOs.

We believe the major clinical application of this LC-MS/MS-based peptide assay would in fact be for pharmacodynamic monitoring. The decreased levels of the peptide in CSF upon SOD1 ASO treatment are indicative of reduced SOD1 protein levels in the CNS of the treated SOD1-mutant rats. The reduction also closely follows the reduction in SOD1 mRNA measured in spinal cord tissue, demonstrating its strong potential as a marker to serve in monitoring for target engagement in clinical trials using SOD1-targeted therapies. Based on comparisons with the ELISA for the SOD $1^{\mathrm{G} 93 \mathrm{~A}}$ cohort, it also appeared that the peptide assay is a more sensitive option for pharmacodynamic monitoring in CSF. Though the pretreatment peptide levels may vary by mutation, we believe this is a pharmacodynamic marker that could be used for all SOD1 ALS patients, as long as pretreatment and posttreatment levels are measured for each patient. In addition to ASO-based therapies, we believe the peptide assay may be applicable to other lines of therapy that target SOD1. For example, though we could not perform a similar experiment in rats using Arimoclomol, a drug that coinduces HSPs that affect protein folding, a single patient carrying V148G treated with Arimoclomol did show a nearly 3 -fold lower level of the peptide concentration as compared with 2 presymptomatic (and untreated) V148G carriers (Table 1), suggesting a pharmacodynamic potential for SOD1-targeting drugs that affect misfolding and stability.

Altogether, we believe this finding demonstrates a new approach for stratifying differences in stability and degradation of diseased proteins that have peptide products released into the extracellular fluid. This particular assay provides a highly sensitive snapshot of differences in SOD1 processing, even when intracellular levels of full-length SOD1 protein remain indistinguishable. Such an approach may be well suited for targeting of protein degradation products in other neurological disorders associated with protein misfolding, such as $\alpha$-synuclein peptide products in the affected subset of Parkinson's patients.

\section{Methods}

Human sample acquisition. All patient samples were obtained from previously collected CSF, stored in 1 of 3 repositories: (a) NEALS, (b) Washington University in St. Louis, and (c) University of Miami Clinical Research in ALS, from IRB-approved studies. Sample identities (mutation type or control) from repositories 2 and 3 were blinded before assay and data sharing. UCSD Biochemical Genetics laboratory was approved by UCSD IRB for analyzing previously collected samples. 
A

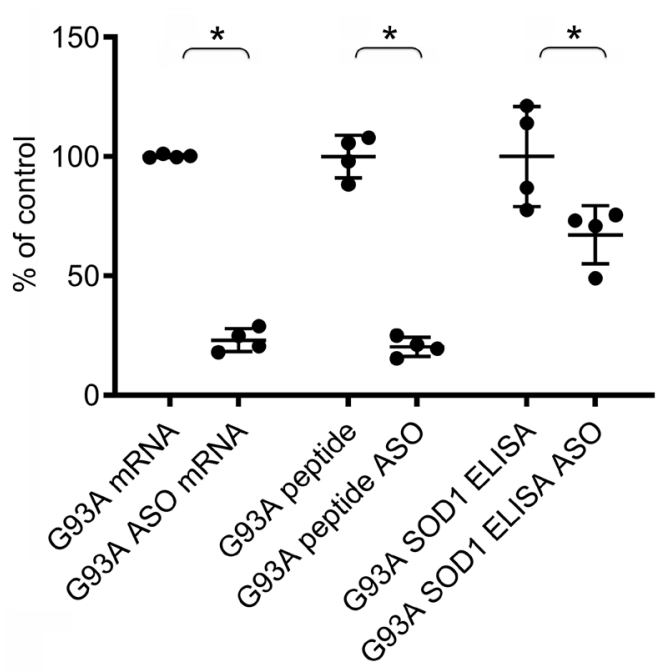

B

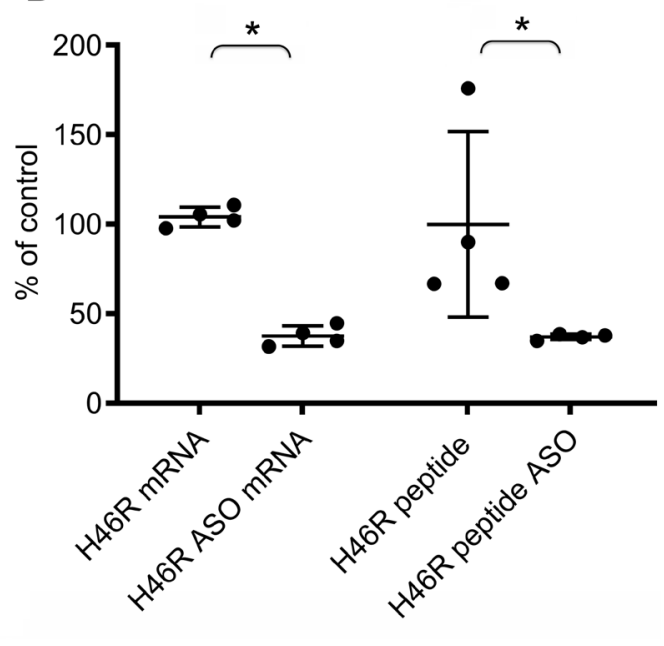

Figure 4. Effects of SOD1-targeted ASO treatment on levels of mRNA, SOD1 peptide (LC-MS/MS), and SOD1 protein (ELISA). mRNA was measured in spinal cords of control and ASO-treated rats, while SOD1 peptide and protein assays were performed on CSF samples from the same rats. (A) Presymptomatic SOD1 ${ }^{C 93 A}$ rats $(n=4: 2$ saline injected, 2 noninjected) compared with ASO-treated littermates $(n=4)$. For the mRNA assay, LC-MS/MS assay, and ELISA, 77\%, $80 \%$, and $32 \%$ mean reductions were measured, respectively, following ASO treatment. (B) Presymptomatic SOD1 ${ }^{146 R}$ rats ( $n$ $=4: 2$ saline injected, 2 noninjected) compared with ASO-treated littermates $(n=4)$ showing $64 \%$ and $63 \%$ reduction in mean mRNA and peptide levels, respectively. ELISA data for SOD1 ${ }^{\text {H46R }}$ were inconclusive as described in the Results section and are not shown. ${ }^{*} P<0.05$ using Mann-Whitney $U$ tests for all comparisons.

Peptide identification. Regarding MS/MS, initial peptides of interest were searched in silico for potential sequence matches using Protein Prospector software (UCSF). Two potential sequences matched nearly all experimental fragment ions, and these 2 along with 3 others with slightly worse overlap were custom-synthesized for confirmation (GenScript Corp.). Each of the 5 peptides was evaluated for chromatographic elution time, peak shape, and MS/MS fragmentation patterns and compared with the experimental peptide of interest. Two of these had identical elution times as the compound of interest, but only 1 of these, SDGPVKV, exactly matched the chromatographic and fragmentation data (MS/MS), as shown in Supplemental Figure 1.

Peptide quantification. Following discovery of the peptide marker from untargeted metabolomics platform (described in Supplemental Methods), $100 \mu 1$ of each CSF samples was extracted with $400 \mu$ l of ice-cold methanol containing $500 \mathrm{pM}$ of a stable isotope version of the peptide (containing ${ }^{15} \mathrm{~N}_{2} /{ }^{13} \mathrm{C}_{6}$ lysine, synthesized by Sigma Aqua peptides). A 7-point standard curve (using $100 \mu 1$ of analyte-stripped serum as the blank matrix) was prepared with each batch, containing a range of 10 to $1000 \mathrm{pM}$ of unlabeled peptide standard for the bulk of data collection (an initial range of 100 to $5000 \mathrm{pM}$ was used for the first batch), shown in Supplemental Figure 5. Following vortexing for 30 seconds and deproteinization, supernatants were removed and dried in a speed vac (integrated with a lyophilizer for cold drying; Thermo Fisher). The sample was resuspended in 40 $\mu 1$ of acetonitrile $/ 0.1 \%$ formic acid (FA) (Honeywell). Half of the material was loaded as a $20-\mu 1$ injection on a Waters Xbridge C18-BEH column (incubated at $30^{\circ} \mathrm{C}$ in column oven). API 4000 and API 4500 (AB Sciex) instruments were used for data collection and Multiquant software (AB Sciex) was used for quantitation calculations. The MRM transition 351.2>499.3 was used for quantitation of the endogenous peptide (collision energy [CE]: 16), while 351.2>246.2 (CE: 23) was used as a confirmatory transition. 355.2>507.30 was used for the isotopically labeled peptide standard. MRM transition 350.7>499.30 (CE: 17) was used to monitor potential nondeamidated peptide. Universal MS parameters used included DP: 70; EP: 10; CXP: 6.0; CAD: 9.0; CUR: 40; IS voltage: 5500; GS1: 40; GS2: 70; and heating gas of $450^{\circ} \mathrm{C}$. We used 100-ms dwell times for each transition (0.5-second total scan cycle time). Mobile phases included (a) $0.1 \%$ FA in water and (b) $100 \%$ acetonitrile $/ 0.1 \% \mathrm{FA}$. The following gradient steps were used for each 23 -minute run: (a) 0 - to 3-minute isocratic at $95 \% \mathrm{~A}$, (b) 3-8 minutes $(95 \% \mathrm{~A}$ to $50 \% \mathrm{~A}$ ), (c) 8-8.1 minutes $(50 \% \mathrm{~A}$ to $0 \% \mathrm{~A})$, (d) 8.1 - to 13 -minute isocratic at $0 \% \mathrm{~A}$, (e) $13-13.1$ minutes $(0 \% \mathrm{~A}$ to $95 \% \mathrm{~A}$ ), and (f) 13.1 - to 23 -minute isocratic at $95 \% \mathrm{~A}$. LC-MS/ MS-grade water was purchased from Honeywell. 
Table 1. Study participant characteristics and peptide concentrations

\begin{tabular}{|c|c|c|c|c|c|}
\hline Mutation & Group & $\begin{array}{c}\text { Sex (no. F/ } \\
\text { no. M) }\end{array}$ & $\begin{array}{c}\text { Mean age at } \\
\text { collection (years) }\end{array}$ & $\begin{array}{c}\text { Mean disease } \\
\text { duration } \\
\text { (years) }\end{array}$ & $\begin{array}{l}\text { Peptide concentration } \\
\text { (pM) (mean } \pm \text { SD) }\end{array}$ \\
\hline V148G & 2 presymptomatic & $2 / 0$ & 54 & (n/a) & $755.0 \pm 137.2$ \\
\hline V148G & 1 symptomatic, treated $^{\mathrm{A}}$ & $0 / 1$ & $(n / s)$ & 2.5 & 250.0 \\
\hline G41D & 2 presymptomatic & $1 / 1$ & $(n / s)$ & $(n / a)$ & $447.0 \pm 59.4$ \\
\hline G85R & 2 symptomatic & $0 / 2$ & 57 & 1.7 & $302.5 \pm 152.0$ \\
\hline A4T & 2 symptomatic & $0 / 1$ & $(n / s)$ & 1.0 & 270.0 \\
\hline A4V & 5 symptomatic & $2 / 3$ & 48 & 1.1 & $224.6 \pm 43.0$ \\
\hline L144F & 3 presymptomatic & $1 / 2$ & 55 & $(n / a)$ & $160.3 \pm 28.0$ \\
\hline I113T & 5 presymptomatic & $5 / 0$ & 43 & $(n / a)$ & $148.2 \pm 59.8$ \\
\hline С93А & 1 symptomatic & $1 / 0$ & $(n / s)$ & 1.3 & 147.0 \\
\hline A89V & $\begin{array}{l}1 \text { symptomatic, } \\
2 \text { collections }\end{array}$ & $0 / 1$ & $(n / s)$ & 5.3 & $81.1 \pm 0.3$ \\
\hline G935 & 1 symptomatic & $0 / 1$ & $(n / s)$ & 10.0 & 30.7 \\
\hline D90A & $\begin{array}{l}1 \text { presymptomatic/1 } \\
\text { symptomatic }\end{array}$ & $1 / 1$ & 60 & $0.7^{\mathrm{B}}$ & $27.2 \pm 3.6$ \\
\hline G37R & 1 symptomatic & $1 / 0$ & $(n / s)$ & $(n / a)$ & 25.2 \\
\hline Disease controls & 30 neuro. disease ${ }^{\complement}$ & - & - & - & $25.8 \pm 2.8$ \\
\hline Controls & 29 healthy controls ${ }^{\complement}$ & - & - & - & $24.3 \pm 4.8$ \\
\hline \multicolumn{6}{|c|}{$\begin{array}{l}\text { CSF samples were acquired from } 3 \text { repositories: Northeastern ALS Consortium (NEALS), Washington University in St. } \\
\text { Louis, and University of Miami. For genetic variants that had only } 1 \text { sample, to maximize participant anonymity, no age } \\
\text { or sex is displayed. ASample collected after participant was treated with Arimoclomol as part of a clinical trial. }{ }^{\text {BFor the }} \\
\text { symptomatic participant only. 'Sex and age information not available for the control cohorts. (n/a), not applicable (if } \\
\text { presymptomatic) or not available (if symptomatic); (n/s), not shown. }\end{array}$} \\
\hline
\end{tabular}

Interbatch/instrument normalization. The first batch of quantitative analysis was performed on an API 4000 triple quadrupole instrument with a standard curve range of 100 to $5000 \mathrm{pM}$, a range selected based on preliminary measurements. Though most of the SOD1-mutant samples fell well within this range, the control samples and 3 of the SOD1 samples were below the limit of quantitation. All future batches were run with an adjusted standard curve range (10-1000 pM) to cover the full range of peptide levels in controls and patients, also using a more sensitive instrument, the API 4500 (AB Sciex). Several of the control samples and SOD1 samples were rerun in these later batches to help normalize samples from the first batch that were below the levels of accurate quantitation. The majority of all samples, therefore, did not require any further normalization and are expressed in absolute concentrations fit to linear regressions of standard curves shown in Supplemental Figure 5. Many of the samples from the first batch were not simply rerun on the newer platform because of a limited volume of sample that was required for repeat extraction/analysis. Quality controls (QCs) at different concentration ranges were run in multiple batches, but a full bioanalytical validation using 4 consecutive days of repeat standard curve and QC analyses to determine intraday and interday precision and accuracy was not performed for this research study but will be done before use in any future clinical applications.

Immunoblot analysis. Transgenic rats expressing $\mathrm{hSOD} 1^{\mathrm{G} 93 \mathrm{~A}}(34)$ and $\mathrm{hSOD} 1^{\mathrm{H} 46 \mathrm{R}}(35)$ were as originally described and maintained by standard protocols. All animal procedures were consistent with the requirements of the Animal Care and Use Committees of the University of California. Spinal cords from non- $\mathrm{Tg}$ SOD $1^{\mathrm{WT}}$ and symptomatic SOD $1^{\mathrm{G} 93 \mathrm{~A}}$ or SOD1 ${ }^{\mathrm{H} 46 \mathrm{R}}$ rats were homogenized in cold PBS $1 \times$ supplemented with protease inhibitors. The lysates were centrifuged for 10 minutes at $1000 \mathrm{~g}$, and the resulting supernatants (clarified tissue extracts) were analyzed by immunoblotting. Equal protein amounts were separated on SDS-PAGE, transferred to nitrocellulose membranes, and probed with the polyclonal anti-SOD1 (Enzo Life Sciences, ADI-SOD-100, diluted at 1:1000) or monoclonal anti-GAPDH antibodies (Abcam, clone $6 \mathrm{C} 5$, ab8245, 1:10,000) followed by horseradish peroxidase-conjugated secondary antibodies (Jackson ImmunoResearch). Pico ECL (Pierce) was used to detect immunoreactive bands.

Rat sample acquisition and injection of ASO. CSF from rats at early symptomatic stages was acquired for metabolomics and ASO experiments as previously described (36). Spinal cords used for native SOD1 deamidation quantitation were obtained following euthanasia as described previously (22). 
Table 2. Levels of mRNA (from spinal cord), peptide (LC-MS/MS assay of CSF), and SOD1 protein (ELISA of CSF) compared between identical control (saline-injected and untreated) as well as ASO-treated rats

\begin{tabular}{|c|c|c|c|c|c|}
\hline Treatment type & $\begin{array}{c}\text { G93A mRNA } \\
\text { (hS0D1/GAPDH) }\end{array}$ & $\begin{array}{c}\text { H46R mRNA } \\
\text { (hSOD1/GAPDH) }\end{array}$ & G93A peptide (nM) & H46R peptide (nM) & G93A ELISA (ng/ml) \\
\hline Saline & 0.883 & 0.293 & 8.83 & 3.11 & 106.37 \\
\hline Saline & 0.877 & 0.306 & 8.65 & 2.30 & 79.65 \\
\hline Untreated & 0.878 & 0.316 & 7.22 & 6.06 & 112.44 \\
\hline Untreated & 0.891 & 0.332 & 8.02 & 2.31 & 78.67 \\
\hline ASO & 0.254 & 0.095 & 2.05 & 1.27 & 74.10 \\
\hline ASO & 0.159 & 0.117 & 1.26 & 1.31 & 49.17 \\
\hline ASO & 0.180 & 0.105 & 1.60 & 1.33 & 69.06 \\
\hline ASO & 0.219 & 0.134 & 1.73 & 1.21 & 67.12 \\
\hline Control: mean \pm SD & $0.882 \pm 0.006$ & $0.312 \pm 0.016$ & $8.18 \pm 0.73$ & $3.45 \pm 1.78$ & $94.28 \pm 17.64$ \\
\hline ASO-treated: mean \pm SD & $0.203 \pm 0.042$ & $0.113 \pm 0.017$ & $1.66 \pm 0.33$ & $1.28 \pm 0.06$ & $64.36 \pm 10.76$ \\
\hline
\end{tabular}

Intracerebroventricular stereotactic injections of $20 \mu \mathrm{l}$ of ASO solution, corresponding to a total of $1000 \mu \mathrm{g}$ of ASO (Ionis Pharmaceuticals), were administered using the following coordinates: $-1.4 \mathrm{~mm}$ $\mathrm{M} / \mathrm{L},-0.4 \mathrm{~mm} \mathrm{~A} / \mathrm{P}$, and $-3.5 \mathrm{~mm} \mathrm{D} / \mathrm{V}$ from the bregma. Presymptomatic (2-month-old SOD1 ${ }^{\mathrm{G} 93 \mathrm{~A}}$ and 4-month-old SOD ${ }^{\mathrm{H} 46 \mathrm{R}}$ ) rats were treated with either PBS $1 \times$ or ASO targeting the human SOD1 sequence (TTAATGTTTATCAGGAT). After 6 weeks of treatment, CSF was collected. In brief, after anesthesia (ketamine/xylazine or propoflo injection), a blunt dissection was made through the musculature down to the atlanto-occipital membrane, between the caudal part of the cerebellum and medulla oblongata, to gain access through to the cisterna magna. CSF was sampled out via 25-gauge needle/syringe aspiration. About $100 \mu 1$ was collected per rat and directly snap-frozen into liquid nitrogen.

$m R N A$ measurement in rat spinal cord from ASO experiment. Levels of hSOD1 mRNA and GAPDH were measured from frozen, powderized spinal cords as performed previously (37). Primer sequences for hSOD1 and rat GAPDH were as follows: hSOD1, forward, TGCATCATTGGCCGCA; reverse, TTTCTTCATTTCCACCTTTGCC; and probe, ACTGGTGGTCCATGAAAAAGCAGATGACTT; GAPDH, forward, TGTTCTAGAGACAGCCGCATCTT; reverse, CACCGACCTTCACCATCTTGT; probe, TTGTGCAGTGCCAGCCTCGTCTCA.

Proteomics experiments are detailed in the Supplemental Methods.

Statistics. Markerview software (AB Sciex) was used for untargeted metabolomics data processing and initial statistical analysis. All further statistical analyses ( $U$ tests, box plots, Spearman's rank correlation analysis, and ROC curves) were performed using GraphPad Prism v.7.01 software. D'Agostino-Pearson normality test was performed to evaluate peptide concentration distributions of both control and SOD1 populations. The control group showed a normal distribution $(P>0.05)$ of peptide concentrations, but the SOD1 group did not. Therefore, nonparametric analyses were used, including Mann-Whitney unpaired $U$ tests for univariate testing $(P<0.05$ considered significant, 2 -tailed) and Spearman's rank correlation analysis.

Peptide concentrations were correlated with previously published stability data of SOD1 using Graph$\mathrm{Pad}$ Prism. The $\Delta \Delta \mathrm{G}$ fit best to the log of the peptide concentration in our analysis. We believe the basis for the scaling can be traced to the standard equation $(\Delta \mathrm{G}=-\mathrm{RT} \operatorname{lnK})$, which can be used to relate the change in protein stability upon unfolding $\left(\Delta \mathrm{G}_{\mathrm{U}}\right)$ with the natural log of the equilibrium constant Keq, composed of the quotient $\mathrm{Ku} / \mathrm{Kf}$ (unfolded/folded).

Study approval. All peptide assays using previously collected human CSF samples was approved by UCSD IRB (La Jolla, California, USA). Patient consent was not required as all samples were acquired from biorepositories previously approved for sharing samples for ALS biomarker studies. All rat experiments were approved by UCSD IUCAC board (La Jolla, California, USA).

\section{Author contributions}

IG contributed to the full study design, development of the peptide quantitation assay, performance of metabolomics and proteomics experiments, interpretation of data, and writing of the manuscript. JW contributed to the human study design, data interpretation, and writing of the manuscript. MMD performed the in vivo ASO experiments. MG contributed to proteomics experiments. KL contributed to the acquisi- 
tion and analysis of the mRNA data. FR and FB contributed to key reagents. MB contributed to human study design, data interpretation, and writing of the manuscript. TMM contributed to the human study design, ASO experimental design, data interpretation, and writing of the manuscript. SDC contributed to the full study design, data interpretation, design of the ASO experiment, and writing of the manuscript.

\section{Acknowledgments}

This work was funded by the NIH, National Institute of Neurological Disorders and Stroke (R21NS099766 to IG, R01NS097816 and R01NS078398 to TM). We thank the NEALS repository for CSF samples. We thank Don Cleveland and John Ravits for thoughtful insight and Ted Hyman for sample blinding and organization.

Address correspondence to: Ilya Gertsman, 7917 Ostrow St., San Diego, California 92111, USA. Phone: 619.543.5279; Email: ilya@clarusanalytical.com.

1. Rosen DR, et al. Mutations in $\mathrm{Cu} / \mathrm{Zn}$ superoxide dismutase gene are associated with familial amyotrophic lateral sclerosis. Nature. 1993;362(6415):59-62.

2. Renton AE, et al. A hexanucleotide repeat expansion in C9ORF72 is the cause of chromosome 9p21-linked ALS-FTD. Neuron. 2011;72(2):257-268

3. DeJesus-Hernandez M, et al. Expanded GGGGCC hexanucleotide repeat in noncoding region of C9ORF72 causes chromosome 9p-linked FTD and ALS. Neuron. 2011;72(2):245-256.

4. Kabashi E, et al. TARDBP mutations in individuals with sporadic and familial amyotrophic lateral sclerosis. Nat Genet. 2008;40(5):572-574

5. Sreedharan J, et al. TDP-43 mutations in familial and sporadic amyotrophic lateral sclerosis. Science. 2008;319(5870):1668-1672.

6. Vance C, et al. Mutations in FUS, an RNA processing protein, cause familial amyotrophic lateral sclerosis type 6 . Science. 2009;323(5918):1208-1211.

7. Kwiatkowski TJ, et al. Mutations in the FUS/TLS gene on chromosome 16 cause familial amyotrophic lateral sclerosis. Science. 2009;323(5918):1205-1208.

8. Andersen PM. Amyotrophic lateral sclerosis associated with mutations in the CuZn superoxide dismutase gene. Curr Neurol Neurosci Rep. 2006;6(1):37-46.

9. Andersen PM, Al-Chalabi A. Clinical genetics of amyotrophic lateral sclerosis: what do we really know? Nat Rev Neurol. 2011;7(11):603-615.

10. Wang Q, Johnson JL, Agar NY, Agar JN. Protein aggregation and protein instability govern familial amyotrophic lateral sclerosis patient survival. PLoS Biol. 2008;6(7):e170.

11. Martinez A, Palomo Ruiz MD, Perez DI, Gil C. Drugs in clinical development for the treatment of amyotrophic lateral sclerosis. Expert Opin Investig Drugs. 2017;26(4):403-414.

12. Smith RA, et al. Antisense oligonucleotide therapy for neurodegenerative disease. J Clin Invest. 2006;116(8):2290-2296.

13. Miller TM, et al. An antisense oligonucleotide against SOD1 delivered intrathecally for patients with SOD1 familial amyotrophic lateral sclerosis: a phase 1, randomised, first-in-man study. Lancet Neurol. 2013;12(5):435-442.

14. McCampbell A, et al. Antisense oligonucleotides extend survival and reverse decrement in muscle response in ALS models. $J$ Clin Invest. 2018;128(8):3558-3567.

15. van Zundert B, Brown RH. Silencing strategies for therapy of SOD1-mediated ALS. Neurosci Lett. 2017;636:32-39.

16. Vígh L, et al. Bimoclomol: a nontoxic, hydroxylamine derivative with stress protein-inducing activity and cytoprotective effects. Nat Med. 1997;3(10):1150-1154.

17. Kalmar B, Novoselov S, Gray A, Cheetham ME, Margulis B, Greensmith L. Late stage treatment with arimoclomol delays disease progression and prevents protein aggregation in the SOD1 mouse model of ALS. J Neurochem. 2008;107(2):339-350.

18. Kieran D, Kalmar B, Dick JR, Riddoch-Contreras J, Burnstock G, Greensmith L. Treatment with arimoclomol, a coinducer of heat shock proteins, delays disease progression in ALS mice. Nat Med. 2004;10(4):402-405.

19. Phukan J. Arimoclomol, a coinducer of heat shock proteins for the potential treatment of amyotrophic lateral sclerosis. IDrugs. 2010;13(7):482-496.

20. Benatar M, et al. Randomized, double-blind, placebo-controlled trial of arimoclomol in rapidly progressive. Neurology. 2018;90(7):e565-e574.

21. Winer L, et al. SOD1 in cerebral spinal fluid as a pharmacodynamic marker for antisense oligonucleotide therapy. JAMA Neurol. 2013;70(2):201-207.

22. Vande Velde C, Miller TM, Cashman NR, Cleveland DW. Selective association of misfolded ALS-linked mutant SOD1 with the cytoplasmic face of mitochondria. Proc Natl Acad Sci U S A. 2008;105(10):4022-4027.

23. Riemenschneider M, Lautenschlager N, Wagenpfeil S, Diehl J, Drzezga A, Kurz A. Cerebrospinal fluid tau and $\beta$-amyloid 42 proteins identify Alzheimer disease in subjects with mild cognitive impairment. Arch Neurol. 2002;59(11):1729-1734.

24. Gertsman I, Gangoiti JA, Barshop BA. Validation of a dual LC-HRMS platform for clinical metabolic diagnosis in serum, bridging quantitative analysis and untargeted metabolomics. Metabolomics. 2014;10(2):312-323.

25. Robinson NE. Protein deamidation. Proc Natl Acad Sci U S A. 2002;99(8):5283-5288.

26. Tyler-Cross R, Schirch V. Effects of amino acid sequence, buffers, and ionic strength on the rate and mechanism of deamidation of asparagine residues in small peptides. J Biol Chem. 1991;266(33):22549-22556.

27. Nielsen M, Lundegaard C, Lund O, Keşmir C. The role of the proteasome in generating cytotoxic T-cell epitopes: insights 
obtained from improved predictions of proteasomal cleavage. Immunogenetics. 2005;57(1-2):33-41.

28. Di Noto L, Whitson LJ, Cao X, Hart PJ, Levine RL. Proteasomal degradation of mutant superoxide dismutases linked to amyotrophic lateral sclerosis. J Biol Chem. 2005;280(48):39907-39913.

29. Lindberg MJ, Byström R, Boknäs N, Andersen PM, Oliveberg M. Systematically perturbed folding patterns of amyotrophic lateral sclerosis (ALS)-associated SOD1 mutants. Proc Natl Acad Sci U S A. 2005;102(28):9754-9759.

30. Kabuta T, Suzuki Y, Wada K. Degradation of amyotrophic lateral sclerosis-linked mutant $\mathrm{Cu}, \mathrm{Zn}$-superoxide dismutase proteins by macroautophagy and the proteasome. J Biol Chem. 2006;281(41):30524-30533.

31. Johnston JA, Dalton MJ, Gurney ME, Kopito RR. Formation of high molecular weight complexes of mutant Cu, Zn-superoxide dismutase in a mouse model for familial amyotrophic lateral sclerosis. Proc Natl Acad Sci U S A. 2000;97(23):12571-12576.

32. Farr GW, Ying Z, Fenton WA, Horwich AL. Hydrogen-deuterium exchange in vivo to measure turnover of an ALS-associated mutant SOD1 protein in spinal cord of mice. Protein Sci. 2011;20(10):1692-1696.

33. Crisp MJ, et al. In vivo kinetic approach reveals slow SOD1 turnover in the CNS. J Clin Invest. 2015;125(7):2772-2780.

34. Howland DS, et al. Focal loss of the glutamate transporter EAAT2 in a transgenic rat model of SOD1 mutant-mediated amyotrophic lateral sclerosis (ALS). Proc Natl Acad Sci U S A. 2002;99(3):1604-1609.

35. Nagai M, et al. Rats expressing human cytosolic copper-zinc superoxide dismutase transgenes with amyotrophic lateral sclerosis: associated mutations develop motor neuron disease. J Neuroscience. 2001;21(23):9246-9254.

36. Frankmann SP. A technique for repeated sampling of CSF from the anesthetized rat. Physiol Behav. 1986;37(3):489-493.

37. Ling KK, et al. Antisense-mediated reduction of EphA4 in the adult CNS does not improve the function of mice with amyotrophic lateral sclerosis. Neurobiol Dis. 2018;114:174-183. 